Check for updates

Cite this: RSC Adv., 2018, 8, 36712

Received 10th July 2018

Accepted 21st October 2018

DOI: $10.1039 / c 8 r a 05898 a$

rsc.li/rsc-advances

\section{Hybrid-silica nanoparticles as a delivery system of the natural biocide carvacrol}

\begin{abstract}
Chana G. Sokolik (D) and Jean-Paul Lellouche (D) *
Bacterial resistance to common antibiotics necessitates innovative solutions. The phenolic antimicrobial compound carvacrol, a major ingredient in the Essential Oils (EOs) of oregano and thyme, has the advantages of natural compounds such as Generally Recognized As Safe (GRAS) status, but needs an appropriate delivery system designed to overcome its drawbacks (such as low aqueous solubility, easy phenol oxidation, heat/light inactivation, distinct odor). An alkoxysilane incorporating the carvacrol moiety is synthesized and subsequently employed to fabricate hybrid silica nanoparticles (NPs) with carvacrol covalently bound to the silica matrix. The enzymatically hydrolyzable carbamate bond turns these NPs into a release-on-demand nanoscale system for the biocide carvacrol. Characterization of both silane linker and hybrid silica NPs, including quantification of the bioactive compound in the bulk and on the NP surface, is accomplished by spectroscopic methods, including X-ray Photoelectron

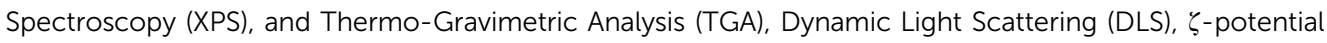
measurements, as well as electron microscopy. Preliminary biological testing with $E$. coli proves an antibacterial effect. The carbamoylation reaction employed to synthesize the hybrid silica precursor might be readily applied to other bioactive phenolic compounds.
\end{abstract}

\section{Introduction}

The significance of antibacterial agents is not limited to strictly pharmaceutical applications for treating and preventing infectious diseases. Many consumer products such as food, cosmetics, and cleaning materials also contain antibacterial agents as standard ingredients to prolong shelf life, maintain freshness, control foodborne pathogens, promote hygiene, and more. However, the widespread use of antibiotic agents leads to emergence of resistant strains of bacteria. Today, there are strains of bacteria that are practically resistant to all common antibiotics. The development of new antibiotics is an extremely expensive enterprise with a very lengthy approval process. Therefore, the World Health Organization warns that we may be heading towards an era in which common infections and minor injuries can kill again. ${ }^{1}$

Antibacterial substances are found in nature in many plants, though, presumably as part of their defense mechanism against harmful microorganisms and reactive oxygen species. One class of such substances is Essential Oils (EOs). Fragrant volatile compounds derived from spice plants compose the EOs. Most EO compounds are terpenes and terpenoids. ${ }^{2}$

EOs and individual EO compounds are attractive candidates for enlisting in the campaign against resistance. Because they are readily available from natural sources, their cost is relatively

Department of Chemistry, Institute of Nanotechnology \& Advanced Materials (BINA), Bar-Ilan University, Ramat-Gan 5290002, Israel.E-mail: lellouj@biu.ac.il low. They have fewer side effects and lower toxicity than nonnatural products and are usually classified as GRAS (Generally Recognized As Safe) by the FDA. ${ }^{3}$ EOs and their compounds have better biodegradability than non-natural antibiotics and preservatives. ${ }^{4}$ The urgency of developing successful EO applications is heightened as concern grows about the safety of using some common broad-spectrum antibiotics, such as triclosan. Triclosan, the use of which was once pervasive in consumer products, was recently banned (among other active ingredients) by the FDA for use in soaps because of potential health risks, including bacterial resistance and hormonal effects. ${ }^{5}$ There are also concerns about its bio-accumulation and toxic byproducts. ${ }^{6,7}$

Because EO compounds affect several cellular targets, they are not likely to induce resistance easily. There also seems to be not much threat of single genetic mutations or series of mutations making the membranes impermeable for EOs because such mutations would probably not allow normal membrane and cell functioning. ${ }^{8}$ Furthermore, several EO compounds may work in synergy with antibiotics through multi-target effects or targeting bacterial resistance mechanisms, for example by inhibiting membranal efflux pumps and protective enzymes. The required dose of antibiotics is then lowered, which can mean that bacteria that had become resistant to a certain antibiotic become again susceptible to the same antibiotic when the EO compound is also present. ${ }^{9-11}$

The mechanism of the antimicrobial effect of EOs is based on their lipophilicity, which enables them to interact with cell 
membranes. The EOs place themselves between the lipid chains, and, as a result, the membranes become more fluid. ${ }^{\mathbf{1 2 , 1 3}}$ This increases the membrane's permeability and disrupts cell homeostasis. As a result, the $\mathrm{pH}$ in the cell and the membrane potential are reduced, which impacts many cell processes, such as ATP formation., ${ }^{2,12}$ Specifically for carvacrol, active ion transport through the membrane via the hydroxyl group has also been proposed. ${ }^{13}$ In addition, research suggests that EOs, among them carvacrol, can interfere with biofilm formation ${ }^{\mathbf{1 4 , 1 5}}$ and inhibit growth of existing biofilms. ${ }^{14}$

Besides antibacterial activity, EOs also exhibit other promising qualities. Specifically, they show anti-inflammatory, ${ }^{4}$ antioxidant, ${ }^{\mathbf{4} 16,17}$ antiradical, ${ }^{16}$ anti-mutagenic, ${ }^{2}$ anti-angiogenic, ${ }^{17,18}$ anti-carcinogenic, ${ }^{4,19,20}$ antiviral, ${ }^{21}$ antimycotic, ${ }^{22-25}$ antiparasitic, ${ }^{26,27}$ and insecticidal activities..$^{28,29}$

However, alongside their advantages, all EO compounds present significant challenges for antibacterial applications. They easily evaporate and, due to their hydrophobicity, have low solubility in aqueous media. In addition, they are susceptible to degradation through exposure to oxygen, UV light, moisture, and heat. To overcome some or all of these problems, two main approaches have been followed: (a) forming nano- and microemulsions of EO compounds or (b) encapsulating EO compounds in (modified) biopolymers, ${ }^{30-33}$ sol-gel materials, ${ }^{34,35}$ or other porous materials such as MOFs (metalorganic frameworks). ${ }^{36}$ In both of these approaches, a system is formed that non-covalently incorporates the EO compound within a matrix. Regarding covalent attachment, eugenol and carvacrol-related aldehydes have been grafted onto chitosan NPs. ${ }^{37}$ Also, eugenol, thymol, and carvacrol have been grafted onto silica microparticles via aldehyde derivatization. ${ }^{38}$

Encapsulation based on inorganic silica makes use of the special properties of silica and the advantages of sol-gel synthesis as a fabrication method. Amorphous silica is regarded as nontoxic, biocompatible, and biodegradable. ${ }^{39}$ Therefore, its use is already established in many applications. ${ }^{\mathbf{4 0 , 4 1}}$ The mild conditions of sol-gel synthesis preserve the full activity of the encapsulated active compound. On the other hand, the inorganic silica matrix offers protection from degrading factors, such as UV light and oxygen, often making the active compound more effective than the free compound. Sol-gel synthesis is a simple, low-cost, and easily scaled-up process. EO-derived compounds and EOs have been encapsulated within silica pores or within inclusion complexes (for example of $\beta$-cyclodextrin) that are in turn encapsulated within a silica phase. ${ }^{42}$ However, because the EO compound is not bound covalently, it may leak out of the pores prematurely and too fast. We, therefore, were interested in developing a silicabased delivery system in which the EO compound is covalently bound and released only when needed, through exposure to bacterial enzymes. This minimal release leads to a sustained antibacterial effect and to significant odormasking (which is an important advantage in many applications involving EOs when the typically strong odor might be undesirable).

For the EO compound in our research, we chose the phenolic carvacrol because of its relatively high antibacterial activity. It is classified by the FDA as Generally Recognized As Safe (GRAS). ${ }^{3,43}$ It can comprise up to $80 \%$ of oregano oil and $2-11 \%$ of thyme oil, the exact amount depending on various factors. ${ }^{12}$ Concerning synthetic covalent derivatization, its phenolic hydroxyl group enables easy synthesis of an organofunctional alkoxysilane that can undergo co-condensation to form hybrid silica materials (see Fig. 1).

\section{Experimental}

\section{Chemicals and reagents}

Carvacrol ( $\geq 98 \%$, FCC, FG), toluene (99.8\%, anhydr.), ammonium hydroxide (ACS reagent, 28.0-30.0\%), tetraethyl orthosilicate (TEOS) (99.999\%) and 3-(triethoxysilyl)propyl isocyanate (95\%) were purchased from Sigma-Aldrich, tetraoctyltin (for synthesis) from Merck and ethanol for synthesis from Bio-Lab (abs., AR). All were used without further purification.

1.
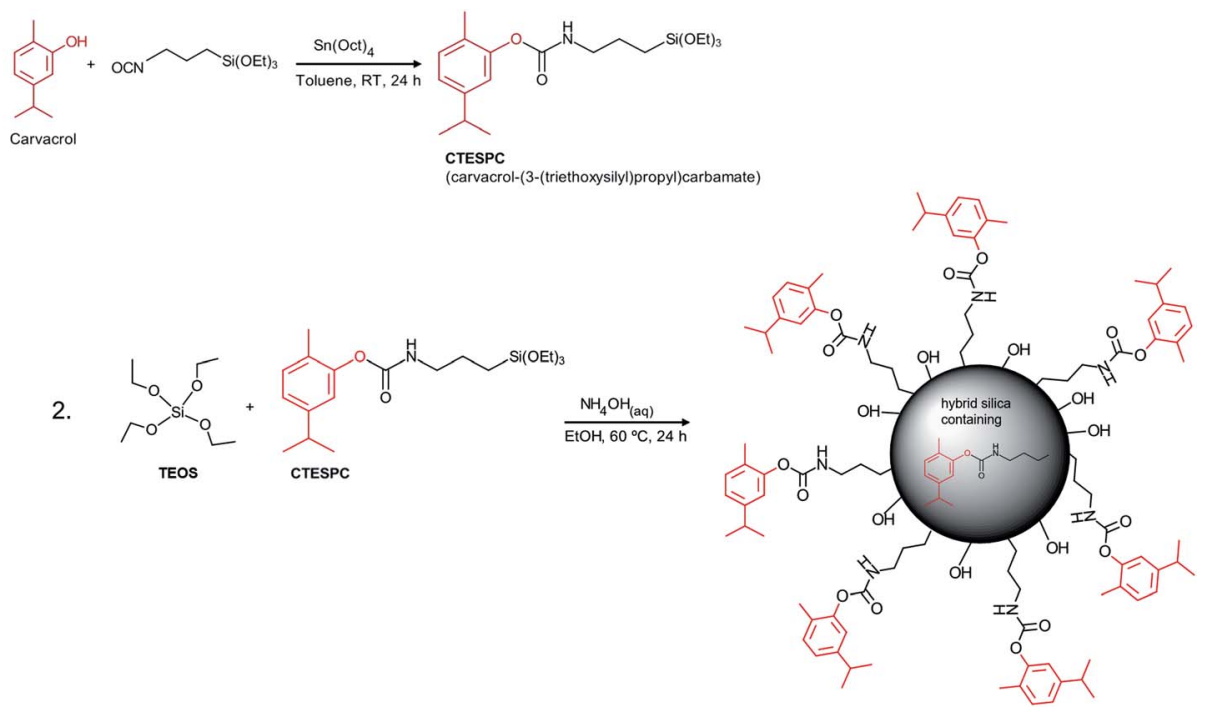

Fig. 1 Reaction scheme of (1) CTESPC synthesis and (2) hybrid silica NP fabrication. 


\section{Synthesis of carvacrol-(3-(triethoxysilyl)propyl)carbamate} (CTESPC)

Carvacrol $(1.078 \mathrm{ml})(7.00 \mathrm{mmol}=1 \mathrm{eq}$.$) was put into an oven-$ dried 3-necked, round-bottom flask $(100 \mathrm{ml})$ under nitrogen and toluene anhydr. $(10 \mathrm{ml})$ was added (yielding a $0.7 \mathrm{M}$ solution of carvacrol). Stirring was started and 3-(triethoxysilyl) propyl isocyanate $(2.600 \mathrm{ml}, 10.5 \mathrm{mmol}=1.5 \mathrm{eq}$. $)$ and tetraoctyltin $\left(\mathrm{CH}_{3}\left(\mathrm{CH}_{2}\right)_{74} \mathrm{Sn}, 6.124 \mathrm{ml}, 10.5 \mathrm{mmol}=1.5\right.$ eq. $)$ were added. Stirring was continued at room temperature under nitrogen atmosphere for at least 15 hours. A thin-layer chromatography (TLC) plate showed the presence of the product (9:1 $n$-hexane : ethyl acetate (EtOAc), silica plate, $R_{\mathrm{f}}$ of product 0.1). After stripping of toluene the product was separated through silica column chromatography (at first 10:1 n-hexane : EtOAc, after elution of all components of the crude mixture except for the product, $6: 1 n$-hexane : EtOAc and then $4: 1$ until elution of CTESPC).

After stripping of the solvent, a clear oil is obtained. Storage in the desiccator turns it into a waxy solid. Yield is $35-54 \%$.

\section{NMR data of carvacrol-(3-(triethoxysilyl)propyl)carbamate (CTESPC)}

${ }^{1} \mathrm{H}$ NMR (300 MHz, $\mathrm{CDCl}_{3}, 25{ }^{\circ} \mathrm{C}$, tetramethylsilane (TMS) as reference) $\delta=7.11\left(\mathrm{~d}, J=7.8 \mathrm{~Hz}, 1 \mathrm{H},(\mathrm{Me})_{2} \mathrm{CHCCHCH}\right), 6.98(\mathrm{~d}$, $\left.J=7.8 \mathrm{~Hz}, 1 \mathrm{H},(\mathrm{Me})_{2} \mathrm{CHCCHCH}\right), 6.92\left(\mathrm{~s}, 1 \mathrm{H},(\mathrm{Me})_{2^{-}}\right.$ CHCCHC(O)), 5.35 (bt, 1H, NH), 3.84 (q, $J=6.9 \mathrm{~Hz}, 6 \mathrm{H}$, $\mathrm{CH}_{3} \mathrm{CH}_{2} \mathrm{O}$ ), 3.28 (m, 2H, NHCH${ }_{2}$ ), 2.86 (sept, $J=6.9 \mathrm{~Hz}, 1 \mathrm{H}$, $\left.(\mathrm{Me})_{2} \mathrm{CH}\right), 2.17\left(\mathrm{~s}, 3 \mathrm{H}, \mathrm{CHC}\left(\mathrm{CH}_{3}\right)\right), 1.71\left(\mathrm{~m}, 2 \mathrm{H}, \mathrm{NHCH}_{2} \mathrm{CH}_{2}\right)$, $1.24\left(\mathrm{t}, J=6.9 \mathrm{~Hz}, 9 \mathrm{H}, \mathrm{CH}_{3} \mathrm{CH}_{2} \mathrm{O}\right), 1.22(\mathrm{~d}, J=6.9 \mathrm{~Hz}, 6 \mathrm{H}$, $\left.\left(\mathrm{CH}_{3}\right)_{2} \mathrm{CH}\right), 0.69\left(\mathrm{~m}, 2 \mathrm{H}, \mathrm{SiCH}_{2}\right){ }^{13} \mathrm{C} \mathrm{NMR}\left(150.9 \mathrm{MHz}, \mathrm{CDCl}_{3}\right.$, $25{ }^{\circ} \mathrm{C}$, TMS $) \delta=154.6(1 \mathrm{C}, \mathrm{NHC}=\mathrm{O}(\mathrm{O})), 149.4(1 \mathrm{C}, \mathrm{NHC}=\mathrm{O}(\mathrm{O})$ C), 147.9 (1C, (O)CCHC(iPr)), 130.7 (1C, CHC(Me)C), 127.7 (1C, (Me)CCHCHC(iPr)), 123.6 (1C, (Me)CCHCHC(iPr)), 120.2 (1C, (Me)CC(O)CH), 58.5 (3C, $\left.\mathrm{CH}_{3} \mathrm{CH}_{2} \mathrm{O}\right), 43.6$ (1C, $\mathrm{NHCH}_{2}$ ), 33.6 (1C, $\left.(\mathrm{Me})_{2} \mathrm{CH}\right), 23.9\left(1 \mathrm{C}, \mathrm{NHCH}_{2} \mathrm{CH}_{2}\right), 23.2\left(2 \mathrm{C},\left(\mathrm{CH}_{3}\right)_{2} \mathrm{CH}\right), 18.3$ (1C, $\left(C_{3}\right)$ C), 15.7 (3C, $C_{3} \mathrm{CH}_{2} \mathrm{O}$ ), 7.7 (1C, $\mathrm{SiCH}_{2}$ ).

\section{Fabrication of hybrid-silica NPs containing carvacrol by Co- condensation via a modified Stöber method}

The amount of CTESPC in the reaction mixture is written as \% $n / n$ and is calculated according to:

$$
\frac{(\text { Number of moles of CTESPC })}{\text { Number of moles of TEOS }} \times 100
$$

CTESPC was weighed into a $20 \mathrm{ml}$ vial (needed weight calculated according to $\% n / n$ that is desired), and EtOH abs. (10 $\mathrm{ml}$ ) was added. At high $\% n / n$, the resulting solution was put into a preheated shaker bath at $50{ }^{\circ} \mathrm{C}$ for $10 \mathrm{~min}$ to improve solubility. TEOS $(0.400 \mathrm{ml})$ and then $\mathrm{NH}_{4} \mathrm{OH}_{(\mathrm{aq})}(28-30 \%, 0.800$ $\mathrm{ml}$ ) was added with vortex mixing after every addition.

The vial was put on an orbital shaker at RT (250 rpm) or at $\geq 15 \% n / n$ into a shaker bath at $60{ }^{\circ} \mathrm{C}(180-220 \mathrm{rpm})$ for $\sim 24$ hours.

The washing and centrifugation cycles were: 3-4 times $\mathrm{H}_{2} \mathrm{O}$ with $\sim 10 \% \mathrm{EtOH}$ to reduce the $\mathrm{pH}$ and remove some of the unreacted CTESPC, 3 times EtOH to remove remaining CTESPC and TEOS (13 000-13 $500 \mathrm{rpm}, 20 \mathrm{~min}, 3{ }^{\circ} \mathrm{C}$ ). If DLS size and $\zeta$ potential were measured in $\mathrm{H}_{2} \mathrm{O}$, another time washing in $\mathrm{H}_{2} \mathrm{O}$ followed. For biological testing, the NPs were washed another 3 times with $\mathrm{H}_{2} \mathrm{O}$. For analyses that require a dry powder and/or for quantification of the NPs ( $\mathrm{mg}$ dry NPs/ml), the washed NPs were re-dispersed in 1.5-3 $\mathrm{ml} \mathrm{H}_{2} \mathrm{O}$, the dispersion frozen in liquid $\mathrm{N}_{2}$ and lyophilized to dryness (0.001 mbar, 24 hours, $\left.-106^{\circ} \mathrm{C}\right)$.

\section{Fabrication of similar non-hybrid silica NPs by Stöber method as reference for analyses and control in biological testing}

EtOH abs. $(10 \mathrm{ml})$ was put in a vial of $20 \mathrm{ml}$. $\mathrm{NH}_{4} \mathrm{OH}_{(\mathrm{aq})}(28-$ $30 \%, 0.8 \mathrm{ml})$ and TEOS $(0.4 \mathrm{ml})$ were added with vortex mixing after every addition. The mixture was put on an orbital shaker for 24 hours at RT. The resulting NPs were washed 3 times with $\mathrm{H}_{2} \mathrm{O}$ and 3 times with EtOH. The remaining details are identical to those of the hybrid-silica NPs.

\section{Biological testing}

Escherichia coli 8739 were grown for 20-24 hours in Nutrient Broth (NB, Sigma) media under shaking $(250 \mathrm{rpm})$ at $37^{\circ} \mathrm{C}$. The following day, the overnight cultures were diluted in fresh NB medium to obtain a stock solution with a concentration of $50 \times$ $10^{5}$ Colony Forming Units (CFU) per $\mathrm{ml}$ (according to the relation of $\mathrm{OD}_{600}$ of $1=0.8 \times 10^{9} \mathrm{E}$. coli cells common as standard lab practice in microbiology). The stock solution $\left(20 \mu \mathrm{l}, 10^{5} \mathrm{CFU}\right.$ in $20 \mu \mathrm{l})$ was added to the NP solution $(1 \mathrm{ml})$. The resulting solution was incubated at $37{ }^{\circ} \mathrm{C}$ for 24 hours. Then serial dilutions were carried out and the cells spotted in duplicate onto NB agar plates. The NB agar plates were incubated at $37^{\circ} \mathrm{C}$ for $16-$ 20 hours after which the colonies were counted.

The experimental controls were doubly-deionized water and pure silica NP solutions at approx. the same concentration of $\mathrm{mg} \mathrm{NPs} / \mathrm{ml}$ as the tested solution.

Instrumentation. ${ }^{1} \mathrm{H}-\mathrm{NMR}$ spectra were measured on an Avance II Bruker $300 \mathrm{MHz}$ spectrometer at $25^{\circ} \mathrm{C}$ in $\mathrm{CDCl}_{3}$ with use of $\mathrm{Me}_{4} \mathrm{Si}$ (tetramethylsilane, TMS) as internal standard. ${ }^{13} \mathrm{C}$ NMR spectra were measured on an Avance Bruker DMX 600 MHz spectrometer under the same conditions.

Mass spectroscopy using Electrospray Ionization (ESI) was carried out on an Agilent 6100 Single Quad MS system with methanol as solvent.

An FT-IR spectrometer of Thermo-Scientific with iD7 ATR accessory with a diamond crystal was used for characterization of CTESPC at 100 scans, a resolution of $4 \mathrm{~cm}^{-1}$, and a data spacing of $0.482 \mathrm{~cm}^{-1}$.

The hydrodynamic particle diameters, size distributions and $\zeta$-potential of the particles were measured on a Nano-ZS Zetasizer Nano series of Malvern Instruments, Ltd., UK. For particle size, the measurement conditions were $25{ }^{\circ} \mathrm{C}$ (no equilibration time), automatic measurement duration, 3 measurements with up to 12 sub-runs, no delay between measurements and automatic attenuation selection. A disposable cuvette was used. $\zeta$ potential measurements were carried out with a minimum of 10 and a maximum of 100 runs at $25^{\circ} \mathrm{C}$. DTS 1070 folded capillary 
$\zeta$-potential cells were filled with sample dispersions $(0.8 \mathrm{ml})$ : washed NPs were dispersed in EtOH or $\mathrm{H}_{2} \mathrm{O}(1.5-3 \mathrm{ml})$, diluted $1: 1.5$ with EtOH or $\mathrm{H}_{2} \mathrm{O}$, and sonicated for $4 \mathrm{~min}$ in a lowpower sonication bath (Elmasonic S 30 ultrasonic bath, 37 $\mathrm{kHz}$ at full power). For DLS measurements, this solution was diluted 1 : 35 with EtOH or $\mathrm{H}_{2} \mathrm{O}$. For $\zeta$-potential measurements, the ratio was $1: 18$ or $1: 19$. In both cases, an additional sonication of 4 min was carried out right before the measurement.

TEM images were obtained on a JEM-1400, JEOL and Tecnai G2, FEI instrument equipped with a Gatan CCD camera, at an operating voltage of $120 \mathrm{kV}$. For preparation of TEM grids washed NPs were dispersed in EtOH (1.5-3 ml). The resulting suspension was diluted $1: 1.5$ with $\mathrm{EtOH}$ and sonicated for 4 min in a low-power sonication bath (Elmasonic S 30 ultrasonic bath, $37 \mathrm{kHz}$ at full power). After an additional dilution $1: 75$ with EtOH and 4 min sonication in the sonication bath, a drop was placed onto a 400 mesh carbon-covered copper grid and dried in air.

HR-SEM images were obtained on a FEI, Magellan 400L. Powder of dried NPs was distributed on double-sided carbon tape pasted onto a copper grid. Then, carbon coating was applied.

Quantification of carvacrol in the NPs (\% w/w) was carried out through measuring the weight loss as a function of temperature. Measurements were carried out on a TGA/DSC 1 STAR $^{\mathrm{e}}$ System of Mettler Toledo and the conditions employed were a temperature range of $25-800{ }^{\circ} \mathrm{C}$ and a heating rate of $10{ }^{\circ} \mathrm{C} \mathrm{min}{ }^{-1}$. The gas employed was air at a rate of $50 \mathrm{ml} \mathrm{min}^{-1}$. Samples were put into an alumina pan. Since the MW of carvacrol $\left(150.22 \mathrm{~g} \mathrm{~mol}^{-1}\right.$ ) constitutes $64.1 \%$ of the organic part of the CTESPC molecule (see Fig. 2), the weight loss of Step 2 (after the subtraction of the average weight loss of 6 samples of non-hybrid silica NPs in Step 2) is multiplied by 0.641 to obtain an estimate of "mg carvacrol/mg NPs".

For quantification of carvacrol in NPs through UV absorption, lyophilized NPs were suspended in $\mathrm{NH}_{4} \mathrm{OH}_{(\mathrm{aq})}(25-30 \%)$ to a concentration of $0.3-1.3 \mathrm{mg} \mathrm{NPs} / \mathrm{ml} \mathrm{NH}_{4} \mathrm{OH}_{(\mathrm{aq})}$. The suspension was kept in a shaker bath at $60^{\circ} \mathrm{C}$ for 24 hours to dissolve the silica and release the carvacrol. The concentration of carvacrol was then determined through use of a calibration curve of carvacrol in $\mathrm{NH}_{4} \mathrm{OH}_{(\mathrm{aq})}(25-30 \%)$ at $293 \mathrm{~nm}$ (Fig. 3). The measurements were done on a Cary 100 Bio UV-visible

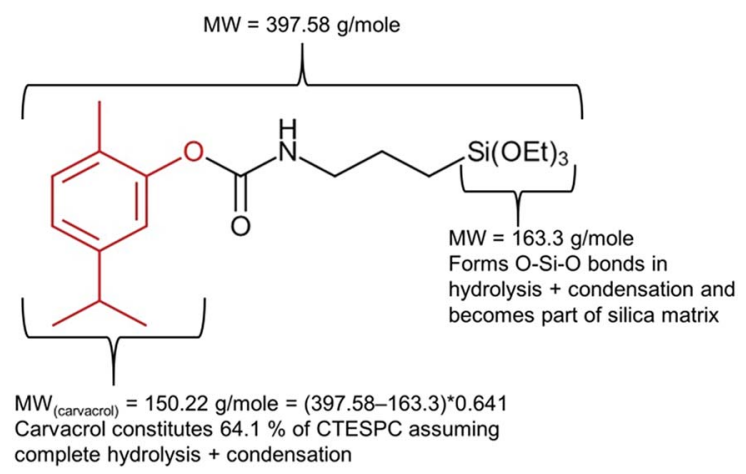

Fig. 2 Explanation of factor 0.641 used in calculation of carvacrol content $\left(\mathrm{mg} \mathrm{ml}^{-1}\right)$ in CTESPC-containing NPs based on TGA results.

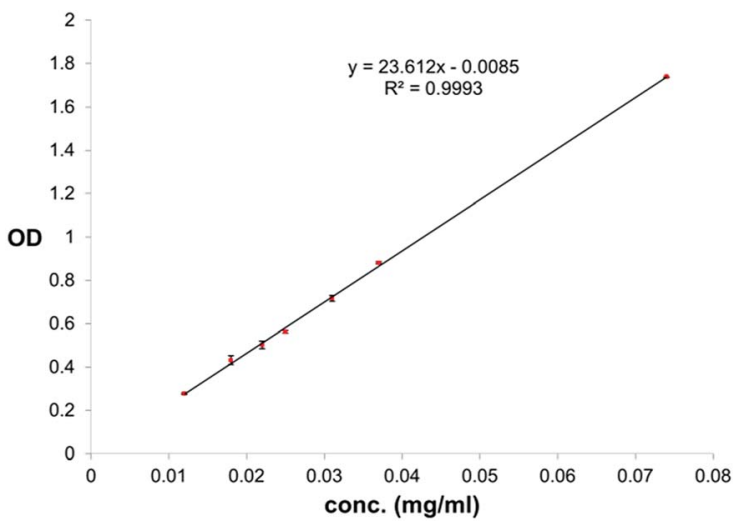

Fig. 3 Calibration curve for quantification of carvacrol in $\mathrm{NH}_{4} \mathrm{OH}_{\text {(aq) }}$ through UV absorption at $293 \mathrm{~nm}$.

spectrophotometer of Varian Inc. The conditions were a scan rate of $600.00 \mathrm{~nm} \mathrm{~min}^{-1}$, an average time of $0.100 \mathrm{~s}$, a data interval of $1.000 \mathrm{~nm}$, in double beam mode.

XPS measurements were performed on a Kratos Axis HS spectrometer equipped with an $\mathrm{Al} \mathrm{K} \alpha \mathrm{X}$-ray radiation source (photon energy of $1486.6 \mathrm{eV}$ ). The pass energy was $80 \mathrm{eV}$ for survey spectra and $40 \mathrm{eV}$ for HR spectra. The source power was 75 or $150 \mathrm{~W}$. The binding energies (BE) of all elements were recalibrated by setting the $\mathrm{CC} / \mathrm{CH}$ component of the $\mathrm{C} 1 \mathrm{~s}$ peak at $285 \mathrm{eV}$. For quantitative analysis high-resolution core-level spectra were used and Shirley background correction performed. Spectra analysis and deconvolution was performed with the Vision Software (Kratos). Overlapping signals were analyzed after deconvolution into Gaussian/Lorentzian-shaped components.

\section{Results and discussion}

\section{Synthesis of carvacrol-(3-(triethoxysilyl)propyl)carbamate (CTESPC)}

Carvacrol possesses a phenolic hydroxyl group, enabling silanization through formation of a urethane (carbamate) linkage with a silanated isocyanate (in this case 3-(triethoxysilyl)-propyl isocyanate). An efficient catalyst for urethane formation is tetraoctyltin, a Lewis acid catalyst forming a catalytically active complex with alcohols and isocyanates, both of which are electron donors. The bulky octyl substituents seem to depress complexation of the resulting carbamate with the electronaccepting tin atoms, which would reduce the concentration of active catalyst. ${ }^{44}$ Previously, this tetraoctyltin-catalyzed carbamoylation reaction has been utilized to silanate the biocide triclosan. ${ }^{45}$

We confirmed the formation of CTESPC (silanated carvacrol) through ${ }^{1} \mathrm{H}$-NMR, ${ }^{13} \mathrm{C}-\mathrm{NMR}$, Fourier-Transform Infrared (FTIR), and UV-VIS analyses, and mass spectroscopy.

The ${ }^{1} \mathrm{H}-\mathrm{NMR}$ spectrum of the product purified through silica column chromatography shows the broad triplet of the carbamate NH-proton at $5.35 \mathrm{ppm}$ and the presence of the methylene and methyl protons of the silane aliphatic groups at 3.84, 3.28, $1.71,1.24$, and $0.69 \mathrm{ppm}$ (TMS as reference) with the correct integrations and multiplicities. The ${ }^{13} \mathrm{C}-\mathrm{NMR}$ spectrum 


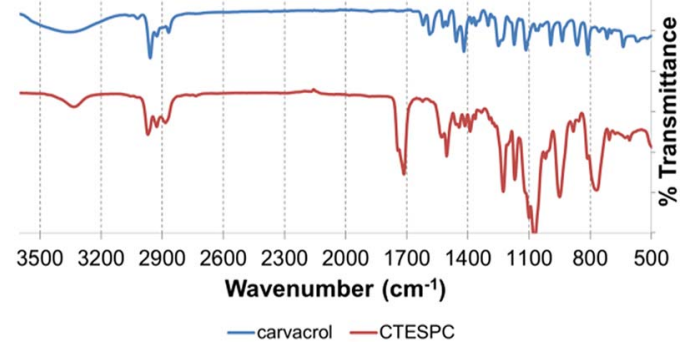

Fig. 4 Comparison of FT-IR spectra of free carvacrol (upper curve) and CTESPC (lower curve).

contains the corresponding carbon peaks of aliphatic carbons at 58.5, 43.6, 23.9, 15.7, and $7.7 \mathrm{ppm}$ (TMS as reference). The DEPT 135 (Distortionless Enhancement by Polarization Transfer) spectrum identifies the peaks at 58.5, 43.6, 23.9, and $7.7 \mathrm{ppm}$ as peaks of methylene carbons, which confirms the covalent attachment of the silane moiety originating from the isocyanate.

In the FT-IR spectrum of CTESPC (Fig. 4), the absorption of the $\mathrm{C}=\mathrm{O}$ stretch of the urethane linkage appears at $1714 \mathrm{~cm}^{-1}$ (s) and the $\mathrm{N}-\mathrm{H}$ stretch at $3334 \mathrm{~cm}^{-1}$ (w). Free carvacrol shows a broad peak centered around $3300 \mathrm{~cm}^{-1}$ (s) of the phenolic $\mathrm{OH}^{-}$ stretch with hydrogen-bonding, which does not appear in CTESPC. At $1100 \mathrm{~cm}^{-1}(\mathrm{~m})$ and $1074 \mathrm{~cm}^{-1}(\mathrm{~s})$, there is a strong, broad peak with a typical doublet shape which is also seen in the spectrum of TEOS (not shown), confirming silane formation. According to the wavenumbers, these peaks can be assigned to a Si-O stretch of silicon alkoxides $\left(1000-1100 \mathrm{~cm}^{-1}\right.$ $(\mathrm{s}))^{46}$ or to the $\mathrm{C}-\mathrm{O}$ stretch of the ethoxide group (1050$\left.1175 \mathrm{~cm}^{-1}\right) \cdot{ }^{47}$ Some sources assign $1074 \mathrm{~cm}^{-1}$ to both groups, while some assign both peaks, 1100 and $1074 \mathrm{~cm}^{-1}$, to the $\mathrm{C}-\mathrm{O}$ stretch. ${ }^{48}$ At 813 and $769 \mathrm{~cm}^{-1}(\mathrm{~m})$, we see a similarly-shaped, but weaker peak. In the spectrum of TEOS, there is one peak at $785 \mathrm{~cm}^{-1}$. It is attributed to the $\mathrm{Si}-\mathrm{O}$ or the $\mathrm{C}-\mathrm{O}$ stretch. ${ }^{48}$ The broader shape with the double peak here might be caused by the additional absorption of the Si-C stretch $\left(700-820 \mathrm{~cm}^{-1}\right.$ $(\mathrm{m}))^{46}$ which is not found in TEOS.

The mass spectrum in electrospray ionization shows a strong peak at $\mathrm{m} / \mathrm{z}$ of 420.2 , belonging to $\mathbf{M}^{+}+\mathrm{Na}$. It confirms the molecular weight, which is calculated as $397.58 \mathrm{amu}$.

The UV absorption spectrum (Fig. 5) shows a shift of the plateau at $218 \mathrm{~nm}$ (free carvacrol) to $211 \mathrm{~nm}$ (CTESPC) and the local absorption maximum at $276 \mathrm{~nm}$ to $\sim 265 \mathrm{~nm}$, which confirms covalent modification of carvacrol.

\section{Optimization of fabrication of NPs with covalently-bound carvacrol - TEM and DLS diameter and $\zeta$-potential}

When the NPs are in the presence of pathogens, the bacterial membrane esterases can hydrolyze the $\mathrm{C}-\mathrm{O}$ single bond of the urethane group of the NPs. Then, carvacrol located on the surface of the particles is released. For that purpose, the NPs need to be in contact with the bacterial cell membrane. This contact increases interaction between enzymes and the NP surface, beyond what is possible by diffusion alone. To

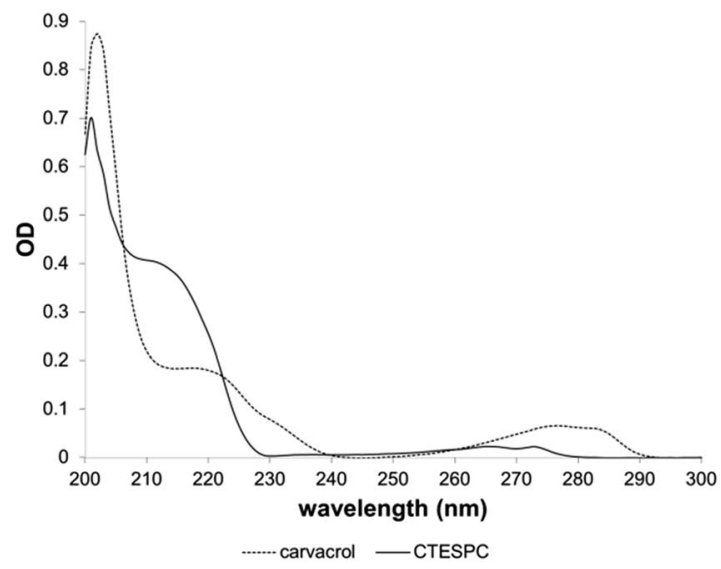

Fig. 5 Comparison of UV absorption spectrum of carvacrol and CTESPC.

maximize contact, the NPs should be stable and not too large because aggregation and large size reduce the NP surface area. Because EO compounds have an MBC (minimum bactericidal concentration) that is by several orders of magnitude higher than the MBC of conventional antibiotic agents, the concentration of CTESPC on the NP surface needs to be high. However, a high concentration of organic content affects the NPs' properties.

With large amount of carvacrol in the NPs in $\% \mathrm{w} / \mathrm{w}$, the Transmission Electron Microscopy (TEM) size decreases to below $50 \mathrm{~nm}$ (Fig. 6A). CTESPC, especially the isopropyl group, seems to cause steric hindrance of the reactive sites for condensation that prevents further NP growth and leads to enrichment of the NP surface in CTESPC, which is desirable. This is also evident from the fact that $\zeta$-potential decreases with increasing $\% \mathrm{w} / \mathrm{w}$ of carvacrol (Fig. 6B) because the density of charged silanol groups on the surface decreases. The downside of this is that low $\zeta$ potential means lower electrostatic repulsion and the NPs are more prone to aggregate. The organic groups on the NP surface form van der Waals, hydrophobic, and $\pi$-stacking interactions with organic groups on other NPs. Aggregation manifests itself in large DLS size (Fig. 6C) and large standard deviation at $>10 \% \mathrm{w} / \mathrm{w}$ carvacrol. An additional reason for the observed changes may be the decrease in $\mathrm{H}_{2} \mathrm{O}$ : $\mathrm{Si}$ and $\mathrm{NH}_{4} \mathrm{OH}$ : Si ratios with increasing CTESPC concentration in the reaction mixture that affect the degree of hydrolysis and condensation. Also, CTESPC possibly undergoes less complete hydrolysis than TEOS.

High CTESPC concentration leads to phase separation in the reaction mixture at RT due to super-saturation of the solution. We, therefore, performed co-condensation at $60{ }^{\circ} \mathrm{C}$.

CTESPC concentration $\geq 35 \% n / n$ (see Experimental) leads to low yield of NPs expressed as $\mathrm{mg} \mathrm{NPs} / \mathrm{ml}$, and at CTESPC concentrations $\leq 35 \% \mathrm{n} / \mathrm{n}$ the maximum $\% \mathrm{w} / \mathrm{w}$ of carvacrol in the NPs is on average reached from $17-20 \% n / n$ on. Further increase in CTESPC concentration in the reaction mixture does not lead to significant increase in the amount of CTESPC incorporated in the NPs. On the contrary, it can lead to a slight decrease in CTESPC content. This state of saturation is seen in Fig. 8, which shows the TG curves of several samples of hybrid- 

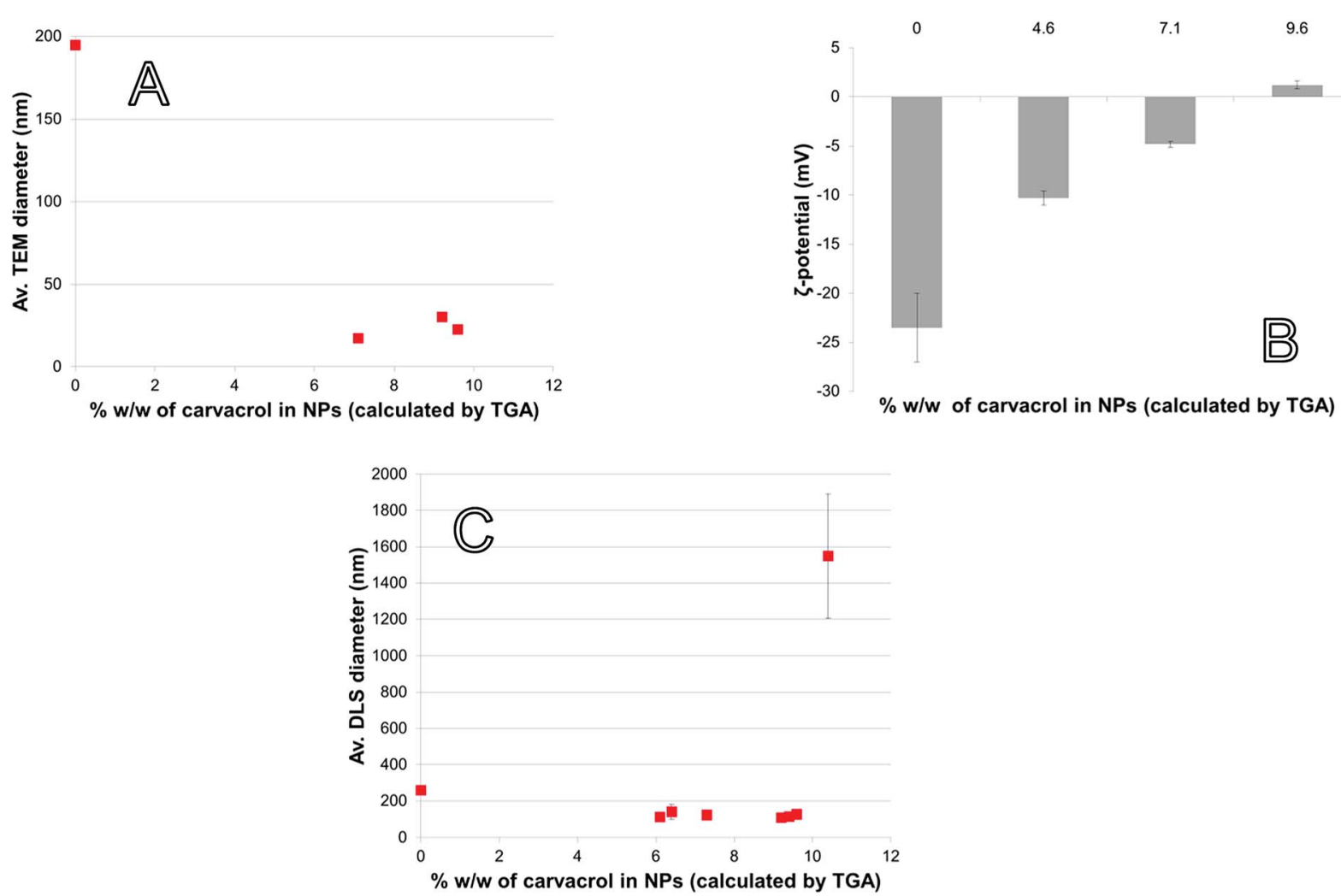

Fig. 6 NP properties as a function of carvacrol content: (A) TEM diameter, (B) ఢ-potential (measured in EtOH), (C) DLS diameter (measured in EtOH).

silica NPs fabricated with high concentration of CTESPC in the reaction mixture. Therefore, $17-20 \% n / n$ CTESPC was chosen as optimal concentration.

\section{Quantification of carvacrol in NPs and on NP surface - TGA/} DSC and XPS

TGA can be used to quantify organic content in an inorganic matrix that does not undergo pyrolysis or combustion in the range of temperatures employed. In the case of hybrid-silica materials, quantification is complicated by the presence of organic content from incomplete hydrolysis in addition to the organic moiety that we intend to quantify. In addition, decrease in weight from adsorbed water and solvent needs to be distinguished from weight loss due to the organic content. Therefore, a thorough analysis of the corresponding weight loss curves is required to correctly estimate the weight loss attributed to the organo-silicate component. Fig. 8 confirms the hydrophobic organic nature of the NP surface because the initial weight loss
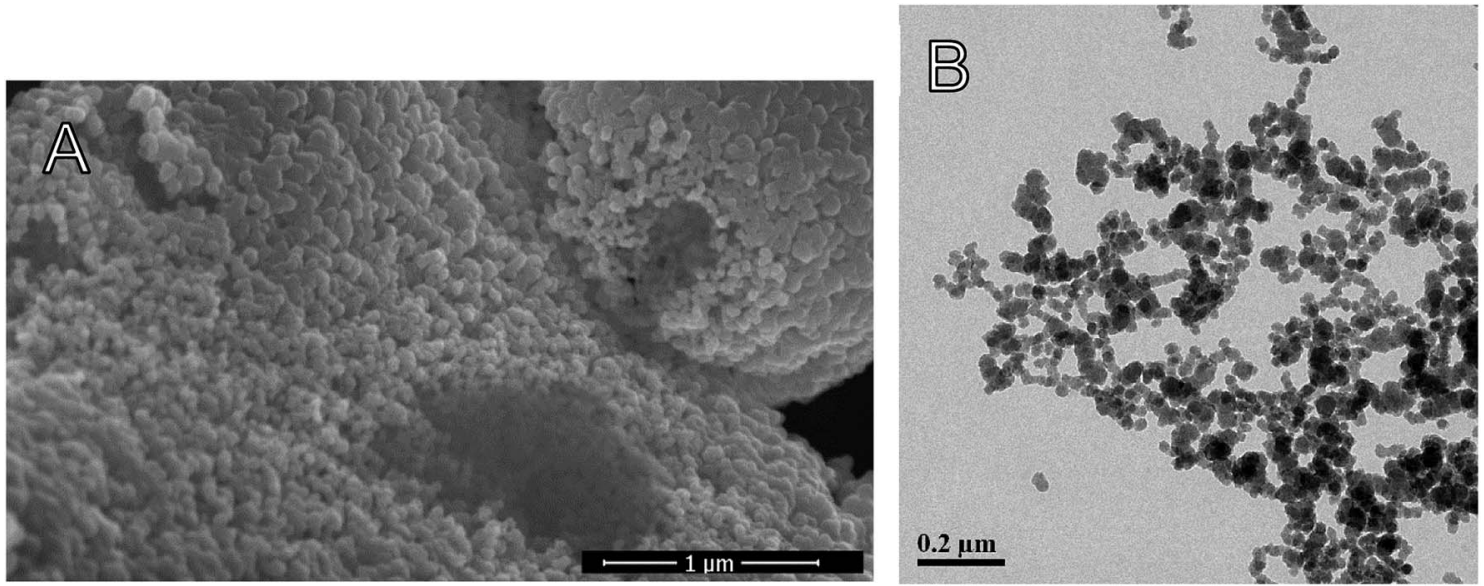

Fig. 7 (A) High Resolution Scanning Electron Microscopy (HR-SEM) of dried CTESPC-containing NPs (fabricated at 25\% $n / n, \sim 10.4 \%$ w/w carvacrol) (scale bar $1 \mu \mathrm{m}$ ), (B) TEM of CTESPC-containing NPs (fabricated at $17 \% n / n, \sim 9.6 \% \mathrm{w} / \mathrm{w}$ carvacrol, av. diameter in TEM $22.9 \mathrm{~nm}$, DLS diameter $127.8 \pm 21.0 \mathrm{~nm}$ ) (scale bar $0.2 \mu \mathrm{m}$ ). 


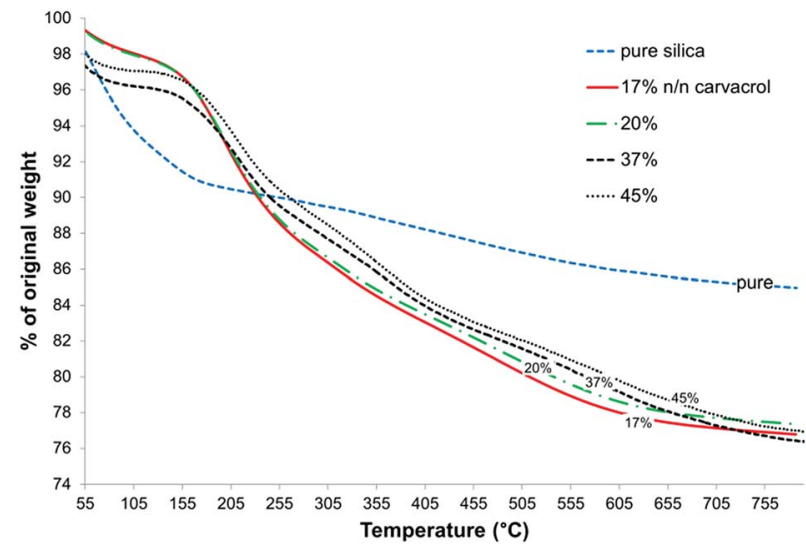

Fig. 8 Comparison of TGA curves of pure silica and of several samples of carvacrol-containing NPs fabricated with different concentrations of CTESPC in the reaction mixture.

due to adsorbed and entrapped water and ethanol is much lower for the carvacrol-containing silica than for pure silica. This is expressed in the flat slope at the lower temperatures.

The first derivative of the TGA curve (called DTG), which expresses the rate of the weight change, was utilized for identification of distinct weight loss events and determining the onset and end of each weight loss event. In addition, integration of the peak area quantifies the weight loss. Fig. 9 shows the DTG curves of non-hybrid (pure) silica NPs and hybrid-silica NPs with high carvacrol content. We can distinguish two major weight loss events. In pure silica NPs, the first event (loss of water and solvent mostly) ends at about $195{ }^{\circ} \mathrm{C}$. It is obvious from the curve that this event constitutes most of the weight loss of silica NPs. For hybrid-silica with high organic content, the first weight loss step ends at $110^{\circ} \mathrm{C}$, and the major weight loss is in this case the second event (the decomposition of the organo-silicate). For quantification of carvacrol in the NPs, we did not differentiate between the weight loss events after the initial one since they all pertain to the decomposition of organic groups. Therefore, we called the range of initial weight loss "Step 1" and the range of the remaining weight loss "Step 2". We subtracted from the weight loss of Step 2 the average weight loss of pure silica NPs in Step 2 , which is probably mostly due to ethoxy groups that did not undergo hydrolysis. This is under the assumption that the average degree of hydrolysis of silica formed from TEOS and hybrid silica formed from TEOS and CTESPC is similar.

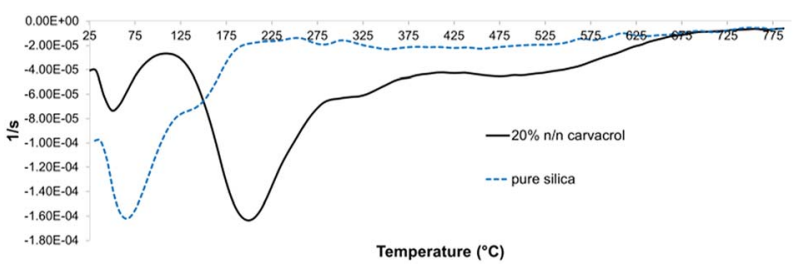

Fig. 9 DTG curves (first derivatives of the TG curves) of pure silica and CTESPC-containing hybrid silica fabricated at $20 \% \mathrm{n} / \mathrm{n}$ CTESPC (the same sample as in Fig. 8).

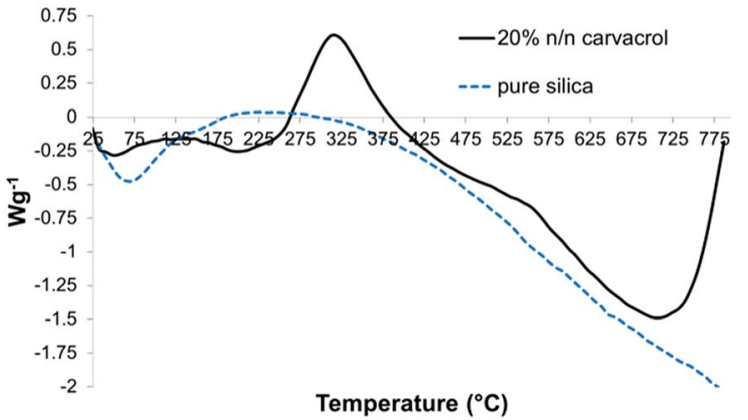

Fig. 10 DSC curves of the same samples as in Fig. 9

The Differential Scanning Calorimetry (DSC) curves served to identify endothermic and exothermic events and their temperature ranges. The DSC curve of hybrid-silica with carvacrol (Fig. 10) shows an exotherm with a maximum at around $325^{\circ} \mathrm{C}$ that is absent in the curve of silica NPs. We assume it corresponds to chemical reactions of the organo-silicate groups while decomposing.

Because only carvacrol that is on the NP surface contributes to the antibacterial effect, it is especially important to characterize the composition of the surface (although it is expected that the composition of the bulk of the NPs reflects the surface composition, too). Therefore, XPS analysis was performed on samples of hybrid silica containing a large percentage of CTESPC. The presence of CTESPC on the surface of the particles was confirmed through the peaks of nitrogen N 1s (BE of 399.80 eV) (Fig. 11B) and the 3 peaks of carbon C 1s (Fig. 11C). The small C 1s peak at highest binding energy (BE: $289.00 \mathrm{eV}$ ) is caused by electron emission from the highly oxidized carbonyl carbon of the urethane linkage. The main peak of $\mathrm{C} 1 \mathrm{~s}$ is asymmetric because it consists of 2 overlapping peaks: the peak of aliphatic and aromatic carbons (BE: $285.01 \mathrm{eV}$ ) and at higher binding energy the peak of the phenolic carbon atom (BE: $286.57 \mathrm{eV}$ ). Peak-fitting was done to deconvolute that peak. These values are in the range of values in the literature: $285 \mathrm{eV}$ for $\mathrm{C} 1 \mathrm{~s}$ of carbon atoms with C-C or C-H bonds, $286.2-288.0 \mathrm{eV}$ for $\mathrm{C} 1 \mathrm{~s}$ of ethers, and $288.0-289.2 \mathrm{eV}$ for C $1 \mathrm{~s}$ peaks of COO. ${ }^{49}$

The ratios of surface atoms in the carvacrol-containing NPs are summarized in Tables 1 and 2 .

To show the atom ratio, the atomic concentration expressed as percentage was divided by the lowest percentage, which was defined as representing one atom and rounded. Table 2 shows that the ratio of the oxidized carbon atoms $(\mathrm{COO}: \mathrm{CO}=1: 1)$ is the correct ratio found in CTESPC.

For each molecule of CTESPC represented by 1 nitrogen, 14 carbon, 1 silicon, and 3.5 oxygen atoms (assuming complete hydrolysis and condensation), there were 4 additional oxygen, 2 carbon, and 4 silicon atoms (see Table 1) which originate approximately from 4 TEOS molecules. That means that for every 4 TEOS molecules bound 1 CTESPC molecule is incorporated. This constitutes a high percentage of CTESPC at the top 5-10 $\mathrm{nm}$. Testing several carvacrol NP samples proved that this result is consistent. 

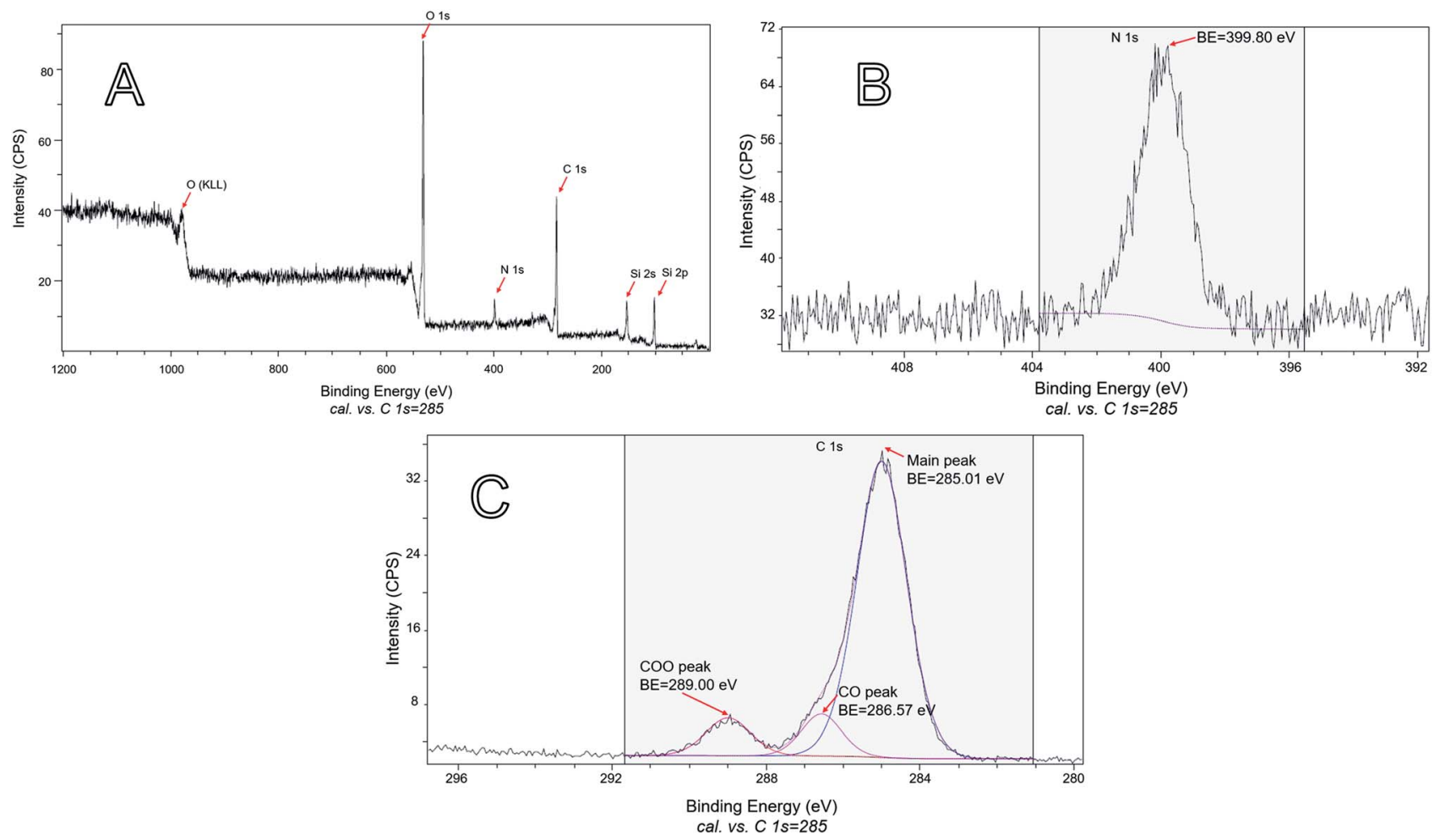

Fig. 11 XPS: (A) survey scan of the sample shown in Fig. 7A. (B) The part of the high-resolution scan that shows the $N$ 1s emission of the sample shown in Fig. 7A. (C) The part of the high-resolution scan that shows the $C$ 1s emissions of the sample shown in Fig. 7A.

Table 1 Quantitative XPS results of all peaks

\begin{tabular}{lclcl}
\hline & $\mathrm{O} 1 \mathrm{~s}$ & $\mathrm{~N} \mathrm{1s}$ & $\mathrm{Si} 2 \mathrm{p}$ & $\mathrm{C} \mathrm{1s}$ \\
\hline \% atomic concentration & 25.61 & 3.43 & 16.93 & 54.03 \\
Atom ratio & 7.5 & 1 & 5 & 16
\end{tabular}

Table 2 Quantitative XPS results of C 1 s peaks

\begin{tabular}{llll}
\hline & C 1s main & C 1s COO & C 1s CO \\
\hline \% atomic concentration & 81.55 & 9.26 & 9.19 \\
Atom ratio & 9 & 1 & 1
\end{tabular}

\section{Preliminary biological results}

We tested the antibacterial activity of the carvacrol-containing hybrid NPs after extensive washing and centrifugation cycles (9-10 cycles, see Experimental) to ensure that no trace of unreacted carvacrol-(3-(triethoxysilyl)propyl)carbamate (CTESPC) was present. E. coli bacteria were incubated for 24 hours with several NP samples fabricated at $\sim 17 \% n / n$ carvacrol whose carvacrol content had been quantified by TGA and UV absorption. It was determined that the concentration required to kill all the bacteria was $1.4 \mathrm{mg} \mathrm{ml}^{-1}$ carvacrol contained in NPs which required a hybrid silica NP concentration in the range of 14$19 \mathrm{mg} \mathrm{ml}^{-1}$ NPs. This seems to be higher than the MBC (minimal bactericidal concentration) of carvacrol in water (determined by us as $0.35 \mathrm{mg} \mathrm{ml}^{-1}$ ), which is comparable to $25 \%$ of the quantity of carvacrol contained in the copolymeric NPs. However, the concentration of carvacrol in the NP solution that is actually active is expected to be lower since all carvacrol is covalently bound and needs to be released by hydrolytic cleavage to become active. This needs close contact between the bacterial cell wall and each NP. Moreover, the copolymeric NPs contain also carvacrol that is not on the surface or is not sterically accessible and therefore is not available for esterases. Pure silica NPs (that is, without carvacrol) showed a certain antibacterial effect, too, but the presence of carvacrol enhances the effect by $\log 4.5$ compared to pure silica, which shows even at slightly higher concentration a much smaller effect, as can be seen in Fig. 12. The advantage of use of the NPs as opposed to pure carvacrol (no

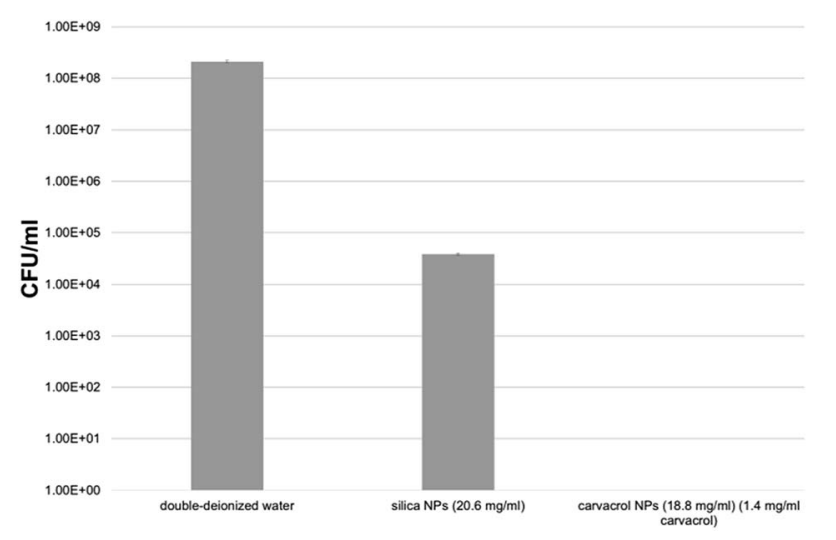

Fig. 12 Results of incubation of CTESPC-containing NPs with E. coli for 24 hours. 
smell and no vapor pressure, low toxicity, sustained release on demand, improved long-term stability and storage properties, better dispersion due to higher hydrophilicity) are discussed in Conclusions.

\section{Conclusions}

We successfully synthesized CTESPC, a silane reagent incorporating the phenolic antibacterial compound carvacrol as organic moiety. We then used CTESPC as precursor for the fabrication of NPs that contain carvacrol covalently-bound with a carbamate bond that is enzymatically hydrolyzable. As a result, the NPs can function as slow-release antibiotics on demand with very little carvacrol released at a time, therefore presumably active for extended periods. Because of the low toxicity of carvacrol and its low concentration due to the slowrelease mechanism further reducing the toxicity, the hybridsilica NPs can be considered a "soft antibiotic". The silica matrix protects the biocide from the degrading effects of UV light, moisture, and exposure to air and heat, which should improve the stability during long-term storage. At the same time the silica matrix also imparts some hydrophilicity to the NPs for their optimal dispersion in water-based media. An important advantage in applications is that the NPs do not contain any unbound carvacrol. Therefore, they have no volatile component and have no smell. This is opposed to the very distinct and strong smell of pure carvacrol in water, which would be undesirable in consumer products and would preclude many applications.

Carvacrol has GRAS status, and the carvacrol used was even FG (Food-Grade). Amorphous silica is also considered safe and nontoxic and is already used in a wide range of consumer applications, including toothpastes, cosmetics, and even food. ${ }^{\mathbf{4 0 , 4 1}}$ Therefore, the antibiotic hybrid-silica NPs that we developed can be used in applications where silica is already present and where an antibacterial effect would be advantageous. Other potential applications include preservatives, dental antibacterial materials, and use in water purification filters.

The one-step carbamoylation reaction that produces CTESPC gives relatively good yields and is easily scaled up. Cocondensation, too, is a simple fabrication method that can easily be adapted to large-scale production and needs no complicated equipment. The antibacterial NPs are expected to be very stable as dry powder or in solution in ethanol or water at neutral pH (which does not encourage spontaneous hydrolysis).

The carbamoylation reaction we employed to synthesize the organo-silane incorporating the antibacterial compound can be readily extended to other phenolic antibacterial compounds, especially other natural terpenoids.

\section{Conflicts of interest}

There are no conflicts to declare.

\section{Notes and references}

1 World Health Organization, Antimicrobial Resistance: Global Report on Surveillance, WHO Press, Geneva, 2014.

2 F. Bakkali, S. Averbeck, D. Averbeck and M. Idaomar, Food Chem. Toxicol., 2008, 46, 446.

3 Food and Drug Administration, CFR - Code of Federal Regulations Title 21, http:/www.accessdata.fda.gov/scripts/ cdrh/cfdocs/cfcfr/CFRSearch.cfm?FR=172.515, accessed, July, 2015.

4 D. Kalemba and A. Kunicka, Curr. Med. Chem., 2003, 10, 813. 5 U.S. Food \& Drug Administration, FDA issues final rule on safety and effectiveness of antibacterial soaps, https:// www.fda.gov/newsevents/newsroom/pressannouncements/ ucm517478.htm, accessed, November, 2017.

6 J. M. Buth, P. O. Steen, C. Sueper, D. Blumentritt, P. J. Vikesland, W. A. Arnold and K. Mc Neill, Environ. Sci. Technol., 2010, 44, 4545.

7 G. S. Dhillon, S. Kaur, R. Pulicharla, S. K. Brar, M. Cledon, M. Verm and R. Y. Surampalli, Int. J. Environ. Res. Public Health, 2015, 5657.

8 K. A. Hammer and C. F. Carson, in Lipids and Essential Oils as Antimicrobial Agents, ed. H. Thormar, John Wiley \& Sons, Ltd, Chichester, UK, 2011, ch. 11.

9 W. T. Langeveld, E. J. A. Veldhuizen and S. A. Burt, Crit. Rev. Microbiol., 2014, 40, 76.

10 P. S. X. Yap, B. C. Yiap, H. C. Ping and S. H. E. Lim, Open Microbiol. J., 2014, 8, 6.

11 K. Palaniappan and R. A. Holley, Int. J. Food Microbiol., 2010, 140, 164.

12 S. Burt, Int. J. Food Microbiol., 2004, 94, 223.

13 A. Ultee, M. H. J. Bennik and R. Moezelaar, Appl. Environ. Microbiol., 2002, 1561.

14 A. Nostro, A. S. Roccaro and G. Bisignano, J. Med. Microbiol., 2007, 519.

15 S. Dalleau, E. Cateau and T. Berges, Int. J. Antimicrob. Agents, 2008, 572.

16 K. Hirasa and M. Takemasa, Spice Science and Technology, Dekker, Inc., New York, 1998.

17 S. P. Piaru, R. Mahmud, A. M. Abdul Majid and Z. D. Mahmoud Nassar, Asian Pac. J. Trop. Med., 2012, 5, 294.

18 W. Chen, M. Gao, J. Wu, A. Wang and R. Shi, J. Ethnopharmacol., 2011, 133, 220.

19 C. B. Yoo, K. T. Han, K. S. Cho, J. Ha, H. J. Park, J. H. Nam, U. H. Kil and K. T. Lee, Cancer Lett., 2005, 225, 41.

20 C. F. Carson and K. A. Hammer, in Lipids and Essential Oils as Antimicrobial Agents, ed. H. Thormar, John Wiley \& Sons, Ltd, Chichester, UK, 2011, ch. 9.

21 C. D. Bishop, J. Essent. Oil Res., 1995, 7, 641.

22 M. A. Azzouz and L. B. Bullerman, J. Food Prot., 1982, 45, 1298.

23 A. Akgul, M. Kivanc and S. Sert, Sci. Aliments, 1991, 11, 361. 24 T. Jayashree and C. Subramanyam, Lett. Appl. Microbiol., 1999, 28, 179.

25 M. Mari, P. Bertolini and G. C. Pratella, ,J. Appl. Microbiol., 2003, 94, 761. 
26 R. Pandey, A. Kalra, S. Tandon, N. Mehrotra, H. N. Singh and S. Kumar, J. Phytopathol., 2000, 148, 501.

27 L. M. Pessoa, S. M. Morais, C. M. L. Bevilaqua and J. H. S. Luciano, Vet. Parasitol., 2002, 109, 59.

28 L. Konstantopoulou, L. Vassilopoulo, P. Mavragani-Tsipidou and Z. G. Scouras, Experientia, 1992, 48, 616.

29 I. Karpouhtsis, E. Pardali, E. Feggou, S. Kokkini, Z. G. Scouras and P. Mavragani-Tsipidou, J. Agric. Food Chem., 1998, 46, 1111.

30 A. Wattanasatcha, S. Rengpipat and S. Wanichwecharungruang, Int. J. Pharm., 2012, 434, 360.

31 B. Shah, P. M. Davidson and Q. Zhong, Int. J. Food Microbiol., 2013, 161, 53.

32 K. Pan, H. Chen, P. M. Davidson and Q. Zhong, J. Agric. Food Chem., 2014, 62, 1649.

33 C. G. Sokolik, R. Ben-Shabat-Binyamini, A. Gedanken and J. P. Lellouche, Ultrason. Sonochem., 2018, 41, 288.

34 M. A. Ashraf, A. M. Khan, M. Ahmad and M. Sarfraz, Front. Chem., 2015, 3, 1.

35 O. Vega, J. J. Araya, M. Chavarria and E. Castellon, J. Sol-Gel Sci. Technol., 2016, 79, 584.

36 L. Paseta, E. Simon-Gaudo, F. Gracia-Gorria and J. Coronas, Chem. Eng. J., 2016, 292, 28.

37 F. Chen, Z. Shi, K. G. Neoh and E. T. Kang, Biotechnol. Bioeng., 2009, 104, 30.

38 M. Ruiz-Rico, E. Pérez-Esteve, A. Bernardos, F. Sancenón, R. Martínez-Máñez, M. Marcos and J. M. Barat, Food Chem., 2017, 233, 228.
39 M. Colilla and M. Vallet-Regi, in Comprehensive Biomaterials, ed. P. Ducheyne, Elsevier, Amsterdam, 2011, vol. 1, ch. 4.429.

40 C. Contada, J. Mejia, O. L. Gracia, J. P. Piret, E. Dumortier, O. Toussaint and S. Lucas, Anal. Bioanal. Chem., 2016, 1, 271.

41 R. Merget, T. Bauer, H. U. Kuepper, S. Philippou, H. D. Bauer, R. Breitstadt and T. Bruening, Arch. Toxicol., 2002, 75, 625.

42 M. Raileanu, L. Todan, M. Voicescu, C. Ciuculescu and M. Maganu, Mater. Sci. Eng., C, 2013, 33, 3281.

43 Food and Drug Administration, Select Committee on GRAS Substances (SCOGS) Opinion: Clove Bud Oil, http:// www.fda.gov/Food/IngredientsPackagingLabeling/GRAS/ SCOGS/ucm261255.htm, accessed, July, 2015.

44 L. Thiele and R. Becker, in Advances in Urethane Science and Technology, ed. K. C. Frisch and D. Klempner, Technomic Publishing, Lancaster, Pennsylvania, 1993, vol. 12, p. 59.

45 I. Makarovsky, Y. Boguslavsky, M. Alesker, J. Lellouche, E. Banin and J. P. Lellouche, Adv. Funct. Mater., 2011, 4295.

46 J. W. Robinson, E. M. Skelly Frame and G. M. Frame, Undergraduate Instrumental Analysis, CRC Press, New York, NY, 2005.

47 P. Yurkanis Bruice, Organic Chemistry, Pearson Education, Inc., Upper Saddle River, NJ, 2007.

48 M. I. Tejedor-Tejedor, L. Paredes and M. A. Anderson, Chem. Mater., 1998, 3410.

49 Database, XPS (X-ray Photoelectron Spectroscopy), XPS Spectra - Chemical Shift/Binding Energy, http:// techdb.podzone.net/xpsstate-e/, accessed, October, 2015. 